\title{
News from the Neuro-Oncology front
}

\author{
Silvia Hofer, Christine Marosi
}

Received: 29 May 2012 / Accepted: 12 June 2012 / Published online: 20 July 2012 (C) Springer-Verlag Wien 2012

Neuro-Oncology, a fast evolving field in oncology, is gaining momentum in the molecular understanding of different brain tumor entities, a fact that might hopefully result in more potent treatment strategies.

A landmark trial of the EORTC in 2005 [1] has set the stage for a widely accepted first line treatment in patients with glioblastoma (GBM, WHO grade IV), comprising maximal safe resection, postoperative radiation with concurrent and adjuvant chemotherapy. Till date, there is no biomarker to guide this standard procedure, although it is well known that GBMs with a methylated MGMT gene promoter are more likely to benefit from combined treatment. Since there is much controversy about the role of biomarkers in Neuro-Oncology, we invited Mathias Preusser to critically review the updated evidence.

What about standard treatment in other gliomas? For anaplastic (WHO grade III) gliomas, there is evidence from three phase III trials [2, 3, 4]. Based on the results of the NOA 04 phase III trial [2], either radiation therapy or chemotherapy seems appropriate as a first line treatment and vice versa for recurrent disease. There is emerging evidence from long-term follow up of two older trials [3, 4] that chemotherapy together with radiation therapy in the upfront setting seems to result in better outcome in LOH 1p,19q co-deleted grade III tumors. Whether first line chemotherapy alone would be an option in this favorable group of patients will have to be demonstrated.

For WHO grade II, also termed low grade gliomas, we are still waiting for the results of a large randomized EORTC trial (22033-26033) in which upfront radiation

\footnotetext{
S. Hofer, MD $(\bowtie)$

Department of Oncology, University Hospital Zurich,

Zurich, Switzerland

e-mail: silvia.hofer@usz.ch

C. Marosi, MD

Department of Oncology, University Hospital Vienna,

Vienna, Austria

e-mail: christine.marosi@meduniwien.ac.at
}

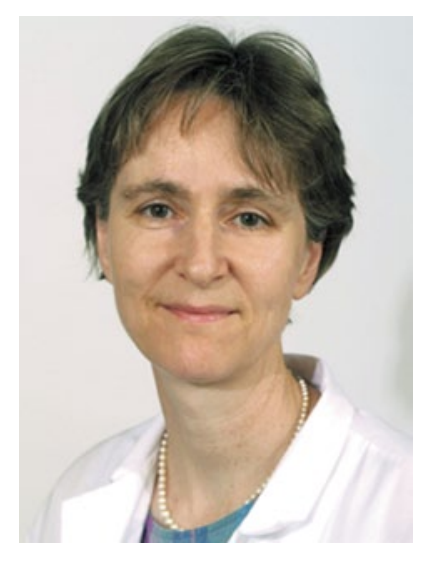

Silvia Hofer

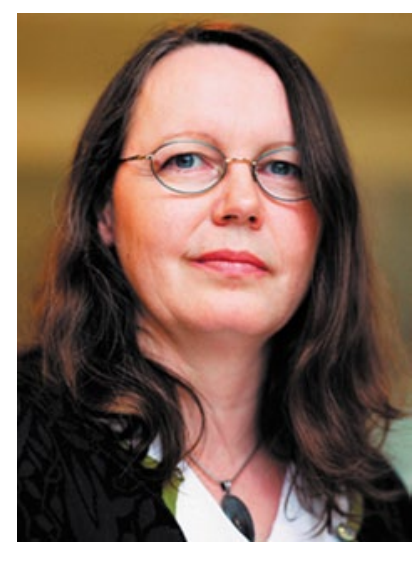

Christine Marosi therapy is compared to chemotherapy in patients with poor prognostic factors. Martin van den Bent shares his comprehensive experience on how to deal best with these tumors whose prognosis is particularly diverse.

There are many other topics in daily Neuro-Oncology practice, for which there is still insufficient data on how best to manage patients. We therefore thought of inviting experts to discuss rare conditions, controversial issues, and novel trends in the management of different primary brain tumors.

Primary CNS lymphomas (PCNSL) were treated for decades with chemotherapy and radiation therapy. A large phase III trial has challenged an old paradigm, particularly about the best time-point of radiation therapy. Lauren Abrey with a long expertise in conducting clinical trials for PCNSL discusses unresolved questions.

Meningiomas are mainly a domain of local therapies. When it comes to locally inaccessible recurrences, systemic treatment might play a role. A review about active drugs is presented by Christine Marosi.

Patients with brain tumors face a limited life expectancy. They and their family members are in need of continuous support, psychological advice, and assistance 
in matters of daily living. End of life (EOL) issues will be covered by Christine Marosi and colleagues.

New treatments need to be monitored and validated by appropriate imaging. There is a broad spectrum of new diagnostic tools, which is presented by Bernhard Schuknecht.

Two rare clinical conditions in Neuro-Oncology are paraneoplastic syndromes and meningeosis carcinomatosa. They are covered by the experts Myrna Rosenfeld and Marc Chamberlain respectively.

Last but not the least, we are much interested in the novel pathways pursued in the management of medulloblastoma(s), tumors that have been lumped together for so many years based on common morphologic features that can now be subclassified and treated accordingly. Stefan Pfister will update us on this topic.

We thank our co-authors for their spontaneous willingness to contribute with their expertise and share with us accumulated knowledge from a multidisciplinary perspective.

\section{Conflict of interest}

The authors declare that there is no conflict of interest in relation to this article.

\section{References}

1. Stupp R, Mason WP, Van Den Bent MJ, Weller M, Fisher B, Taphoorn MJ, et al. Radiotherapy plus concomitant and adjuvant temozolomide for glioblastoma. N Engl J Med. 2005;352(10):987-96.

2. Wick W, Hartmann C, Engel C, Stoffels M, Felsberg J, Stockhammer F, et al. NOA-04 randomized phase III trial of sequential radiochemotherapy of anaplastic glioma with procarbazine, lomustine, and vincristine or temozolomide. J Clin Onol. 2009;27(35):5874-80.

3. Van Den Bent M J, Carpentier AF, Brandes AA, Sanson M, Taphoorn MJ, Bernsen HJ, et al. Adjuvant procarbazine, lomustine, and vincristine improves progression-free survival but not overall survival in newly diagnosed anaplastic oligodendrogliomas and oligoastrocytomas: a randomized european organisation for research and treatment of cancer phase III trial. J Clin Onol. 2006;24:2715-22.

4. Cairncross G, Berkey B, Shaw E, Jenkins R, Scheithauer B, Brachman D, et al. Phase III trial of chemotherapy plus radiotherapy compared with radiotherapy alone for pure and mixedanaplastic oligodendroglioma: Intergroup Radiation Therapy Oncology Group Trial 9402. J Clin Oncol. 2006;24:2707-14. 